\title{
Aspirin versus Indomethacin Treatment of Patent Ductus Arteriosus in Preterm Infants with Respiratory Distress Syndrome
}

\author{
BART VAN OVERMEIRE, FRANK BRUS, KAREL J. VAN ACKER, JEAN C. VAN DER AUWERA, \\ MIEK SCHASFOORT, NYNKE J. ELZENGA, AND ALBERT OKKEN \\ Departments of Paediatrics, Division of Neonatology [B.V.O., K.J.V.A.] and Epidemiology and Community \\ Medicine [J.C.V.D.A.] University Hospital Antwerp, Belgium, and Departments of Neonatology \\ [F.B., A.O.] and Paediatrics, Division of Paediatric Cardiology [M.S., N.J.E.], University \\ Hospital, Groningen, The Netherlands
}

\begin{abstract}
Indomethacin (Indo) is commonly used for treatment of patent ductus arteriosus (PDA) but has renal failure as a main side effect. Aspirin (ASA) is an alternative, but there are no controlled trials on its efficacy. We randomly assigned 75 premature infants suffering from respiratory distress syndrome (RDS) (mean gestational age: $29.6 \pm 2.5 \mathrm{wk}$, mean birth weight: $1295 \pm 464 \mathrm{~g})( \pm \mathrm{SD})$ and on artificial ventilation at the start of the study (mean: $3.4 \mathrm{~d}$ of life), to either Indo $(3 \times 0.2 \mathrm{mg} / \mathrm{kg} / 12$ h) or ASA $(4 \times 15 \mathrm{mg} / \mathrm{kg} / 6 \mathrm{~h})$. PDA and degree of shunting were evaluated by echocardio-Doppler; side effects were carefully recorded. PDA closed in $35 / 38$ patients from the Indo group (92\%) and in 16/37 patients from the ASA group (43\%) ( $p<$ $0.0001)$. Nineteen patients needed further treatment with Indo or surgery (17 in the ASA group and 2 in the Indo group). The only
\end{abstract}

The incidence of PDA in preterm infants with RDS varies between 20 and $60 \%$, depending on the diagnostic criteria that are used and the population that is studied (1-5). Left to right shunting through a PDA is considered to cause several undesirable pulmonary, hemodynamic, renal, and gastrointestinal effects. Therefore, treatment of PDA in RDS patients is indicated before important left to right shunting occurs $(6,7)$.

For many years indomethacin (Indo) has been the drug of choice for the nonsurgical closure of PDA with a reported success rate of $40-80 \%(8,9)$. However, side effects such as oliguria, anuria, and transient renal failure are frequently encountered (10). According to earlier reports ASA also closes the duct effectively (11) and, moreover, has some renal-sparing effect. This sparing effect is probably due to its weaker cyclooxygenase inhibitory effect (12). We wanted to examine whether ASA could induce closure of PDA as sufficiently as Indo and with a less pronounced effect on renal function.

Received October 3, 1994; accepted June 1, 1995

Correspondence: Bart Van Overmeire M.D., Neonatology, University Hospital, Antwerp, Wilrijkstraat 10, B 2650 Edegem, Belgium. side effect observed was a decrease of uresis in the Indo group during $4 \mathrm{~d}$ post treatment $(p<0.01)$. Closing of PDA was positively correlated with gestational age, but not with time of starting Indo/ASA or grade of shunting. We conclude that ASA is not as effective in closing PDA as Indo, but has no adverse effect on uresis. (Pediatr Res 38: 886-891, 1995)

Indo, indomethacin

Abbreviations

ASA, aspirin, acetylsalicylic acid

PDA, patent ductus arteriosus

RDS, respiratory distress syndrome

IPPV, intermittent positive pressure ventilation

CPAP, continuous positive airway pressure
Studies in which ASA and Indo were prospectively compared are not available. We report the results of a prospective randomized study comparing the effectiveness and the side effects of ASA and Indo in the treatment of PDA in neonates with RDS.

\section{METHODS}

\section{Subjects}

The study was conducted in the Neonatal Intensive Care Units of the University of Antwerp, Belgium, and the University of Groningen, The Netherlands. Neonates admitted to both units were eligible for enrollment if the following criteria were met: 1) gestational age below $34 \mathrm{wk}$; 2) treatment with IPPV, CPAP with additional oxygen supply in the inspired air above $30 \%$ or increasing oxygen need requiring CPAP or IPPV because of RDS; 3) postnatal age between 24 and $96 \mathrm{~h}$. Neonates with major congenital malformations including congenital heart defect, persistent pulmonary hypertension of the newborn, or hydrops fetalis, were excluded. 
The daily clinical care of the included patients was performed by attending neonatologists who did not take part in the study. All infants were nursed in humidified incubators during the study period. Fluid intake was primarily guided by body weight, diuresis, and serum sodium concentrations. A weight loss of $10 \%$ was allowed during the first postnatal days. Most patients received one or more transfusions of packed red blood cells or plasma. Ventilated preterm infants with clinical and radiologic signs of RDS received surfactant replacement therapy following the protocol of ongoing clinical trials in both hospitals.

Informed consent was obtained from the parents and the study was approved by the medical ethics committee of both participating hospitals.

\section{Study Protocol}

All patients eligible for enrollment in the study underwent a complete echocardiographic-Doppler evaluation to visualize the PDA and the shunting through it (Vingmed CFM 750 or Hewlett Packard Sonos 1500 with $7.5 \mathrm{MHz}$ transducers; the examinations were recorded on videotape). Shunting through a PDA was graded as follows: 1 ) minor shunting if a small jet was detectable at the pulmonary end of the ductus with colorflow and continuous or pulsed Doppler interrogation, if no disturbed flow was detectable at the level of the pulmonary valves and diastolic forward flow in the pulmonary trunk was absent; 2) moderate shunting if a disturbed diastolic flow was easily detectable at all sites of the pulmonary trunk, a diastolic back flow was present in the aorta immediately beneath the PDA and a forward flow above the PDA; 3 ) severe shunting if a diastolic back flow was detectable in the abdominal aorta at the level of the celiac arterial trunk and if dilatation of the left atrium was present expressed as a left atrium to aortic root ratio above 1.7 on M-mode measurement.

When patients showed marked improvement of their respiratory status on the 2 nd d of life, a delay of $24 \mathrm{~h}$ was allowed to see if IPPV or CPAP could be stopped on the $3 \mathrm{rd} d$ of life. If so, the patient was not entered into the study. If not, the echocardiographic evaluation was repeated; if the patient had PDA he was entered into the study.

Enrolled patients were randomized by sealed envelope technique to receive either Indo treatment (Indocid ${ }^{\circledR}$ ) three times $0.2 \mathrm{mg} / \mathrm{kg}$ i.v. at 12-h intervals, or ASA treatment (Aspegic ${ }^{(B)}$ ) four times $15 \mathrm{mg} / \mathrm{kg}$ i.v. every $6 \mathrm{~h}$. ASA was given i.v. instead of orally, as described in earlier reports (11), to keep bioavailability constant and because most of our patients did not tolerate early gastrointestinal feeding. ASA being a weaker cyclo-oxygenase inhibitor, a relatively high dose was chosen.

If contraindications for treatment with Indo or ASA were present, treatment was either delayed or surgical treatment was applied. These contraindications were: 1 ) recent (less than 48 h) intraventricular hemorrhage; 2) clinical bleeding tendency as revealed by hematuria, blood in the gastric aspirate or in the stools, blood in the endotracheal tube aspirate, oozing from venous or capillary puncture sites; 3 ) thrombocyte count of less than $60000 / \mathrm{mm}^{3}$; 4) oliguria of less than $1 \mathrm{~mL} / \mathrm{kg} / \mathrm{h}$ during the preceding $8 \mathrm{~h} ; 5$ ) blood urea nitrogen in excess of $14 \mathrm{mmol} / \mathrm{L}$ or serum creatinine concentration in excess of $140 \mu \mathrm{mol} / \mathrm{L}$; and 6) hyperbilirubinemia for which exchange transfusion was necessary.

A second echocardiography and Doppler evaluation was performed in all patients after the last dose of the randomized Indo/ASA treatment to evaluate ductal patency and shunting as defined earlier. To avoid registering temporary ductal constriction, this was performed at least $24 \mathrm{~h}$ after the last dose of Indo or ASA. When PDA was still observed, patients were treated with Indo (3 times $0.2 \mathrm{mg} / \mathrm{kg}$ at 12 -h intervals). To detect a possible late reopening of the ductus, a third echo-Doppler evaluation was performed either after the second course of pharmacologic treatment, or 2-3 d after the second echography in patients who received only one treatment.

The following clinical and biologic parameters were registered before and during medical treatment for PDA.

Clinical parameters. Clinical parameters were findings at physical examination at the time of echocardiography, ventilator settings and daily fluid intake and output, daily body weight, results of cranial ultrasound scan. Bleeding tendency was evaluated using the same criteria as explained under contraindications to treatment. Intravenous fluid intake was expressed in milliliters $/ \mathrm{kg}$ of birth weight rather than actual daily weight, to keep the denominator constant when comparing real changes in day by day fluid intake. Similarly, urine output was expressed in milliliters $/ \mathrm{kg}$ of birth weight $/ \mathrm{h}$.

Laboratory parameters. Laboratory parameters were blood urea nitrogen, serum creatinine, serum electrolytes, hematocrit and $\mathrm{Hb}$ concentration, thrombocytes, and cultures when there was suspicion of infection. In a subset of five study patients additional blood samples were collected for serum salicylate concentrations. Samples were taken immediately before and 30 min after each of the four i.v. doses of ASA. The salicylate concentration in serum was measured by gas chromatography after acetylating the extract with acetic anhydride and methylating with diazomethane. In another subset of the study patients who had an indwelling arterial catheter, blood samples were collected for serum thromboxane $\mathrm{B}_{2}$ levels before the first dose of ASA, $30 \mathrm{~min}$ later, and $30 \mathrm{~min}$ after the third dose of ASA. Samples were kept at room temperature for $15 \mathrm{~min}$ to allow clot formation and thereafter centrifuged for $5 \mathrm{~min}$ at $13000 \mathrm{rpm}$. Serum was separated and immediately frozen at minus $20^{\circ} \mathrm{C}$. Thromboxane $\mathrm{B}_{2}$ was measured by RIA.

The outcome. Outcome in the surviving patients was defined on the basis of the need for additional oxygen supply on the 28 th postnatal day.

\section{Statistical Analysis}

It was calculated that a sample size of 35 infants in each group would be necessary to detect with $90 \%$ power at the 0.01 level a difference of efficiency of at least $30 \%$ for ASA versus the assumed efficiency of $80 \%$ for Indo. Ten more patients were added to compensate for possible dropouts. Statistical hypothesis testing involved the use of standard $t$ test and Pearson's $\chi^{2}$ test and, where the $\chi^{2}$ test was not applicable, Fisher's exact test. For analysis involving observations at different time points, standard analysis of variance with re- 
peated measurements was used. Multiple logistic regression was performed as described by Hosmer and Lemeshow (13). Analytical computer software involved CSS/3: STATISTICA ${ }^{(B)}$ (Statsoft) for standard statistical tests, STATXACT ${ }^{\circledR}$ (Cytel) for analysis of contingency tables, $\mathrm{EGRET}^{\circledR}$ (Serc) for multiple logistic regression analysis, and Solo Power Analysis (BMDP) ${ }^{\circledR}$ for sample size and power calculations.

\section{RESULTS}

Patients. Two hundred and twenty patients were eligible for entry into the study and underwent an echocardio-Doppler evaluation between the 2 nd and 4 th $\mathrm{d}$ of life. Seventy-five of them entered the study yielding a power of $92 \%$ in the calculation of the difference in efficiency. The reason for nonentry in the 145 that were excluded was an already closed duct in 106 (47\%), PDA with minor shunting and respiratory improvement in 10 , severe right to left shunting through the ductus due to pulmonary hypertension, thrombocytopenia, and/or recent intraventricular hemorrhage, or sepsis with bleeding tendency in 19 patients. Parental consent was not obtained in another 10 patients.

The studied 75 neonates (39 in Antwerp and 36 in Groningen) were randomized to receive either Indo (38 patients) or ASA (37 patients). The characteristics of the patients are shown in Table 1 ; there were no statistically significant differences between the two groups. A somewhat more marked but still not significant difference was observed for the degree of shunting through the PDA ( $p=0.2$ ). At entry in the study the magnitude of the left to right shunt through the ductus did not correlate with the postnatal age. More patients were treated for PDA on $\mathrm{d} 4$ than on $\mathrm{d} 3$ or 2 . No patients with minor shunting were treated on the 2 nd $d$ of life.

Indo was significantly more efficient than ASA in closing PDA; the closing rate was $92 \%$ in the Indo group versus $43 \%$

Table 1. Patient characteristics after randomization, before first treatment*

\begin{tabular}{lcc}
\hline \multicolumn{1}{c}{ Characteristic } & $\begin{array}{c}\text { Indo } \\
(n=38)\end{array}$ & $\begin{array}{c}\text { ASA } \\
(n=37)\end{array}$ \\
\hline Birth weight $(\mathrm{g})$ & $1292 \pm 434$ & $1298 \pm 494$ \\
Gestational age (wk) & $29.6 \pm 2.5$ & $29.7 \pm 2.5$ \\
Ventilatory support & 36 & 34 \\
$\quad$ IPPV & 2 & 3 \\
CPAP & $43.8 \pm 20.8$ & $44.6 \pm 17.6$ \\
Maximum Fio ${ }_{2} \dagger(\%)$ & $20.9 \pm 3.6 \neq$ & $20.3 \pm 2.99$ \\
Maximum peak pressure $\left(\mathrm{cm} \mathrm{H}_{2} \mathrm{O}\right)$ & 20 & 23 \\
Surfactant administration & & 4 \\
PDA shunting & 2 & 19 \\
$\quad$ Minor & 27 & 14 \\
Moderate & 9 & $3.43 \pm 0.65$ \\
Severe & $3.45 \pm 0.69$ & 2 \\
Start Indo/ASA (d) & & 2 \\
Intraventricular hemorrhage & 2 & 1 \\
Grade 1 & 1 & \\
Grade 2 & & \\
Grade 3-4 & & \\
\hline
\end{tabular}

* Results are expressed in means $\pm S D$ or numbers. No significant differences were observed for any item between the two groups.

$\dagger$ Fraction of inspired oxygen.

$\ddagger n=36$.

II $n=32$. in the ASA group $(p<0.0001)$, a difference of $49 \%(95 \%$ confidence interval, 31-67\%). There was no reopening of the duct after initial closure with the first ASA or Indo treatment.

In 24 patients the ductus arteriosus remained open after the first course of Indo ( 3 patients) or ASA (21 patients). Five of these, one from the Indo group and four from the ASA group, did not receive any further treatment for PDA because of marked improvement of their respiratory status. Nineteen (2 Indo, $17 \mathrm{ASA}$ ) received a second treatment which was Indo in all patients, on average on the $7.7 \pm 2.3( \pm 1 \mathrm{SD}) \mathrm{d}$ of life. The efficiency of this latter treatment was $42 \%$; closure of the PDA occurred in 8 of the 19 patients (Table 2).

Eleven patients (all from ASA group) still had PDA after two courses of medical treatment. Seven of them were treated surgically, three had another course of Indo treatment, and one had been weaned from the ventilator and received no further treatment.

The first $4 \mathrm{~d}$ after either Indo or ASA treatment, uresis was significantly less in the Indo-treated patients than in the ASAtreated patients on $\mathrm{d} 0,1,2$, and $3(p<0.01$ ) (Fig. 1). In the ASA group urine production gradually increased from 3.1 $\mathrm{mL} / \mathrm{kg}$ of birth weight/h on the day before treatment to 4.1 $\mathrm{mL} / \mathrm{kg}$ of birth weight/h on the fifth post treatment day. In the Indo group urine production decreased significantly to a minimal value of $1.9 \mathrm{~mL} / \mathrm{kg}$ of birth weight $/ \mathrm{h}$ on the $2 \mathrm{nd} \mathrm{d}$ after the first dose $(p=0.02)$ and increased again afterward. Daily fluid intake did not differ significantly between both patient groups (Fig. 1); there was a progressive increase in crystalloid fluid intake day by day for both patient groups. The standard deviations are large due to the fact that treatment was started on d 2-4 and that fluid intake was different, depending on birth weight. Patients with birth weights $<1200$ g were given $75-90$ $\mathrm{mL} / \mathrm{kg} / \mathrm{d}$ and patients with larger birth weights were given 60 $\mathrm{mL} / \mathrm{kg} / \mathrm{d}$. Fluid intake was increased with $10 \mathrm{~mL} / \mathrm{kg}$ each day after the first 2-3 postnatal day. Daily weight increase was slight and without significant differences in both treatment groups. No other side effects were observed in both groups, more specifically no increased bleeding tendency, as defined earlier, was seen.

The number of days on ventilation, the number of days of additional oxygen requirements, and the number of patients who developed bronchopulmonary dysplasia (defined as additional oxygen requirement on the 28th postnatal day) did not differ between the two groups. At the age of $28 \mathrm{~d}$, four patients were still on ventilation, and 15 still needed supplemental oxygen in the Indo group versus 6 and 16, respectively, in the ASA group. No significant differences in sepsis or other infectious complications could be demonstrated. There were seven

Table 2. Efficiency of indomethacin treatments*

\begin{tabular}{ccccc} 
& Total & $\begin{array}{c}\text { PDA } \\
\text { closed }\end{array}$ & $\begin{array}{c}\text { PDA } \\
\text { open }\end{array}$ & $\begin{array}{c}\text { Percentage } \\
\text { closed }\end{array}$ \\
\hline $\begin{array}{c}\text { Randomized Indo treatment } \\
\text { (mean age: } 3.5 \mathrm{~d} \text { ) }\end{array}$ & 38 & 35 & 3 & 92 \\
$\begin{array}{c}\text { First nonrandomized Indo } \\
\quad \text { treatment (mean age: } 7.7 \mathrm{~d} \text { ) }\end{array}$ & 19 & 8 & 11 & 42 \\
$\quad$ Total & 57 & 43 & 14 & 75 \\
\hline
\end{tabular}

* Data expressed as numbers and percentages. 

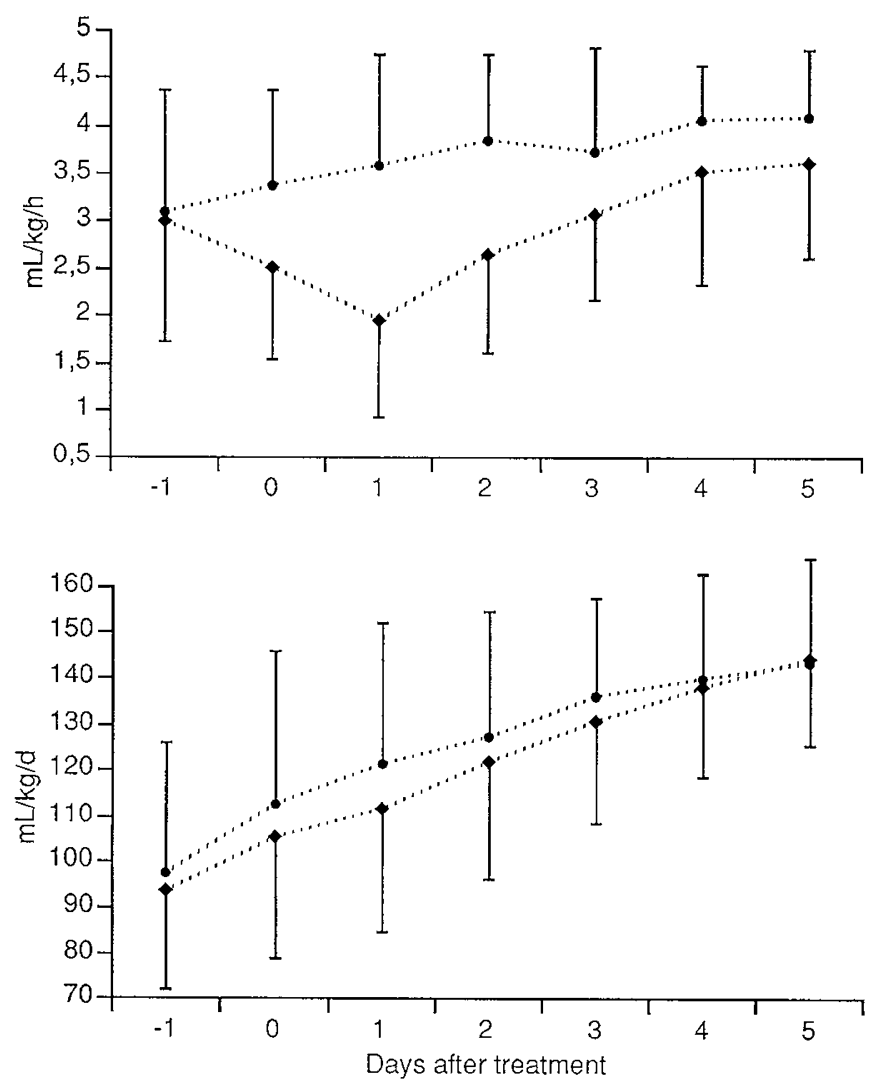

Figure 1. Urine production (upper pannel) and crystalloid fluid intake (lower pannel) in ASA (-)- and Indo ( )-treated patients. Mean \pm 1 SD. Day 0, day of first dose.

neonatal deaths; four in the Indo group (one major intraventricular hemorrhage, overwhelming sepsis in two, intractable respiratory deterioration in one) and three in the ASA group (one major intraventricular hemorrhage, overwhelming sepsis in one, intractable respiratory deterioration in one).

The serum salicylate concentration measured before and after each dose in five patients showed a wide variation but increased after each i.v. dose in each individual patient (Fig. 2). The concentrations decreased in most patients within $6 \mathrm{~h}$ after each dose. In the patient with the highest concentration, the ductus did not close, whereas it did in three others with lower

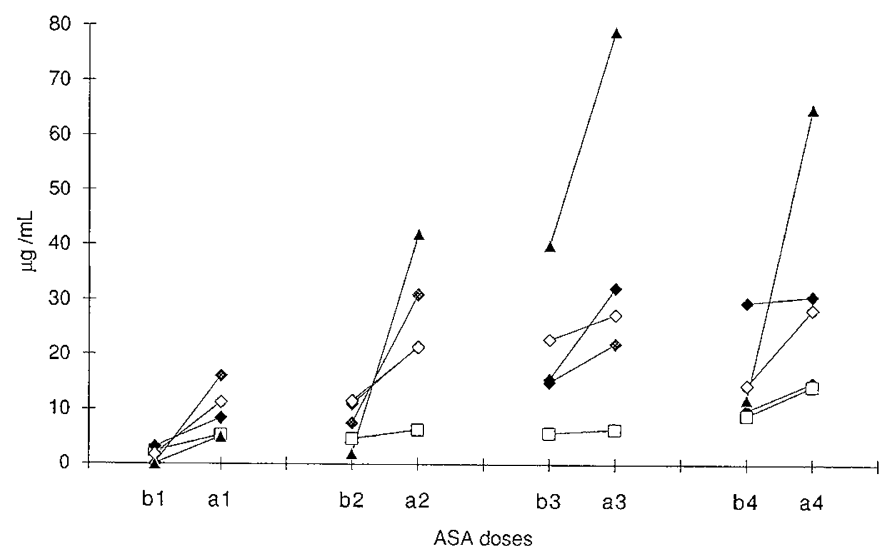

Figure 2. Serum salicylate concentrations before $(b)$ and after $(a)$ each dose in five patients. concentrations: a low concentration therefore did not correspond with persistence of PDA.

The serum thromboxane $\mathrm{B}_{2}$ concentrations fell to significantly lower levels after the first dose of ASA and did not decline much further after the third dose (Fig. 3). Mean values are not shown because the complete successive set of three blood aliquots was obtained in only in three patients. In two of these five patients the ductus was closed after the fourth ASA dose.

To analyze the factors that could predict closure of PDA, 14 parameters were entered in multiple logistic regression analysis (Table 3). In the final model, two parameters showed a significant effect: the choice of Indo versus ASA for first treatment of PDA and the gestational age. Gestational age, within the limits of 25-34 wk in the study population, positively influenced closing of the ductus in both treatment groups (Table 4). There was no correlation between both factors with regard to their predictive power. A larger left to right shunt through PDA and a start of treatment on an earlier postnatal day resulted in higher closure rates in each treatment group; these effects were, however, not statistically significant.

\section{DISCUSSION}

This is the first prospective randomized study which compares the efficacy and the side effects of ASA and Indo for the treatment of PDA. We found that ASA is not as effective as Indo in closing the PDA in preterm infants with RDS. On the other hand, we demonstrated that, as opposed to Indo, ASA treatment was not accompanied by a decrease of uresis.

In comparison with previously reported trials the efficacy of the first course of Indo in the present study is high (92\%). Several factors may have played a role in obtaining this figure. One reason may be that we treated PDA early; the efficacy of Indo has indeed been reported to be inversely related to the postnatal age at the time of treatment, and it has been shown

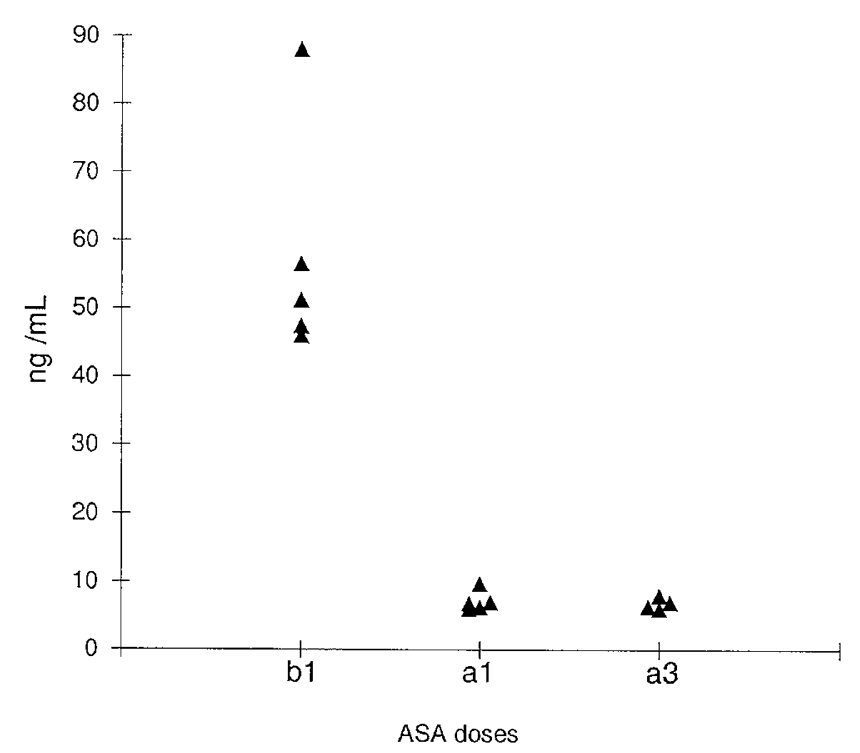

Figure 3. Serum thromboxane $B_{2}$ concentrations before $(b l)$, after the first $(a I)$, and after the third (a3) ASA dose. Successive points do not always represent the same patients. 
Table 3. Clinical parameters evaluated in logistic regression analysis

1. Birthweight
2. Gestational age
3. Sex
4. Weight evolution
5. Fluid intake
6. Uresis
7. Left to right shunt
8. Peak inspiratory pressure
9. Maximal inspired oxygen concentration before treatment
10. Surfactant administration
11. Choice of treatment
12. Age at treatment
13. Total days on ventilation
14. Total days on additional oxygen

Table 4. Predictive factors for closure of PDA*

\begin{tabular}{|c|c|c|c|}
\hline & $\begin{array}{l}\text { Estimated } \\
\text { coefficient }\end{array}$ & $\begin{array}{c}\text { Standard } \\
\text { error of } \\
\text { coefficient }\end{array}$ & $p$ \\
\hline Indo/ASA & -3.79 & 0.966 & $<0.001$ \\
\hline Gestational age & -0.54 & 0.186 & 0.004 \\
\hline Shunt through PDA & 1.02 & 0.637 & 0.111 \\
\hline Time of starting Indo or ASA & 0.91 & 0.628 & 0.152 \\
\hline Constant & 11.25 & 5.56 & 0.043 \\
\hline
\end{tabular}

* Multiple logistic regression model; Log-likelihood $=-25.597$, LR statistic, $5 d f=44.461 ; p<0.001$.

that ducts which are closed during the first $2 \mathrm{~d}$ after delivery have a lower incidence of reopening than those which are treated after the 1 st wk $(14,15)$. Second, our diagnosis of PDA was based on echocardiography and not on clinical findings alone. We adopted this policy because shunting through the ductus may be inapparent clinically but nevertheless may be associated with an increased morbidity and mortality $(16,17)$. Furthermore, a number of treated patients would probably have closed their ducts spontaneously. Spontaneous closure of PDA has been reported in $39-75 \%$ of very low birth weight infants on the 3rd d of life but is unusual in these newborns after that time $(18,19)$. It should also be noted that, when the overall efficacy of Indo in our patients is considered, the figure is much lower: 43 out of 57 treated $(75 \%)$, from which 38 during the first treatment (average age, $3.5 \mathrm{~d}$ ) and 19 during the nonrandomized second treatment (average age, $7.7 \mathrm{~d}$ ) (Table 2). The efficacy of $42 \%$ of the "late" treatment is more comparable with the literature data. Finally, the fluid policy used in all patients resulted in a weight loss after the 2 nd or 3 rd d of life. This additional fluid restriction may also have contributed to the high initial closure rate (15).

The efficacy of ASA in closing PDA in our patients was significantly lower than that of Indo: $43 \%$ (16 with closed PDA out of 37 patients) versus $92 \%$. When we compare this effect of ASA with the spontaneous closure rate after the 4 th $d$ of life in untreated patients, the difference is not significant. Therefore the observed "results" with ASA may reflect spontaneous closure. Another possible explanation for the low efficacy rate of ASA could be that the dosage we used was too low. The dose of $60 \mathrm{mg} / \mathrm{kg}$ of body weight was chosen because 10 $\mathrm{mg} / \mathrm{kg}$ orally, four to six times daily, has been shown to result in sufficient analgesic and anti-inflammatory action in children and infants (20). Furthermore, it has been reported that the newborn eliminates salicylates considerably more slowly than the adult or older child (21); higher dosages could therefore lead to toxic serum concentrations. No data are, however, available on salicylate pharmacokinetics in preterm neonates. When compared with the Indo dose of $0.2 \mathrm{mg} / \mathrm{kg}$ every $12 \mathrm{~h}$, which is only a fraction of the adult dose, $60 \mathrm{mg} / \mathrm{kg}$ ASA is a relatively high dose. The possibility that the low efficacy rate of ASA was due to low dosage is therefore unlikely. Weak cyclo-oxygenase inhibition despite high doses seems more likely. We observed an increase of the serum salicylate levels after each dose and a decline within $6 \mathrm{~h}$ (Fig. 3). These levels may not represent true acetyl salicylate concentrations as the conversion rate of ASA to salicylate in preterm neonates is unknown. We did not investigate the efficacy of a more prolonged ASA treatment or ASA treatment with shorter intervals because of the risk of toxic adverse reactions. There was a definite decline of the serum thromboxane $\mathrm{B}_{2}$ concentrations during ASA treatment. This reflects well the inhibition of cyclo-oxygenase in the platelets. The selective action of ASA on prostaglandin metabolism in the ductus and kidney was not investigated. Provided that conclusions in this respect can be drawn from the study of certain prostaglandin metabolites in serum and urine, it is likely that only the weaker inhibitory action of ASA on cyclo-oxygenase would have been shown, which is already known.

One might argue that even a partial closure of the PDA after ASA treatment could be beneficial clinically, as it results in a diminished shunting. The set up of the protocol, however, did not allow us to study this aspect as almost all infants in whom complete closure of the ductus was not achieved were treated with Indo within an average of $4 \mathrm{~d}$.

The negative effect of Indo therapy on the renal function is well known (10). In contrast with the Indo-treated patients, the patients treated with ASA showed no diminution of diuresis; this can be ascribed to its lower cyclo-oxygenase inhibitory effect.

We did not encounter other side effects, more specifically no increased bleeding tendency during venipuncture or endotracheal aspiration, occult gastrointestinal blood loss, or hematuria. Occurrence of intra- or periventricular hemorrhage or extension of preexisting hemorrhages evaluated by repeated ultrasonic examination of the brain was not observed except in one patient in each group. It has been shown that prenatal maternal aspirin ingestion is associated with abnormal platelet function and increased bleeding tendency in the newborn infant, and its use was associated with intracranial bleeding (22). As it is known that even a single low dose of aspirin can inhibit platelet aggregation for several days (23), and as severe intracranial hemorrhage has been reported even with a lower serum salicylate concentration $(54 \mu \mathrm{L} / \mathrm{mL})(24)$, the absence of these side effects cannot be explained by a low dosage of ASA.

In evaluating the factors which may predict closure of PDA, we observed a significant effect of the gestational age only in the sense that treatment at a lower gestational age resulted in a higher closure rate, independent from the use of either Indo or ASA. There was also an effect of the degree of shunting at the 
start of treatment in the sense that patients with a larger shunt responded better to treatment. This factor, however, did not reach statistical significance.

We conclude from our study that ASA in the relatively high dosage used and under the circumstances of our study design, is less efficient than Indo for early treatment of echocardiographically diagnosed PDA in premature neonates with RDS. Indo appeared to be highly efficient but was accompanied by a significantly brief decrease of the uresis in most patients. In view of these results further studies with ASA, using other treatment schemes, do not seem warranted.

\section{REFERENCES}

1. Dudell GG, Gersony WM 1984 Patent ductus arteriosus in neonates with severe respiratory distress syndrome. J Pediatr 104:915-920

2. Van de Bor M, Verloove-Vanhorick SP, Brand R, Ruys JH 1988 Patent ductus arteriosus in a cohort of 13,338 preterm infants: a collaborative study. Paediatr Perinat Epidemiol 4:328-336

3. Eronen M, Kari A, Pesonen E, Hallman M 1993 The effect of antenatal dexameth asone administration on the fetal and neonatal ductus arteriosus. A randomized double-blind study. Am J Dis Child 147:187-192

4. Zanardo V, Milanesi $O$, Trevisanuto D, Rizzo M, Ronconi M, Stellin G, Cantarutti 1991 Early screening and treatment of "silent" patent ductus arteriosus in prematures with respiratory distress syndrome. J Perinat Med 19:291-295

5. Ellison RC, Peckham GJ, Lang P, Talner NS, Lerer TJ, Lin L, Dooley KJ, Nadas AS 1983 Evaluation of the preterm infant for patent ductus arteriosus. Pediatrics 71:364372

6. Stefano JL, Abbasi S, Pearlman SA, Spear ML, Esterly KL, Bhutani VK 1991 Closure of the ductus arteriosus with indomethacin in ventilated neonates with respiratory distress syndrome; effects on pulmonary compliance and ventilation. Am Rev Respir Dis 143:236-239

7. Meritt TA, Harris JP, Roughmann K 1981 Early closure of the patent ductus arteriosus in very low birth weight infants: a controlled trial. J Pediatr 99:281-286

8. Friedman WF, Hirschklau MJ, Printz MP, Pitlick PT, Kirkpatrick SE 1976 Pharmacologic closure of patent ductus arteriosus in the premature infant. $\mathrm{N}$ Engl $\mathrm{J}$ Med $295: 526-529$
9. Gersony WM, Peckham GJ, Ellison RC, Miettinen OS, Nadas AS 1983 Effects of indomethacin in premature infants with patent ductus arteriosus: results of a national collaborative study. J Pediatr 102:895-906

10. Seyberth HW, Rascher W, Hackenthal R, Wille L 1983 Effect of prolonged indomethacin therapy on renal function and selected vasoactive hormones in very-lowbirth weight infants with symptomatic ductus arteriosus. J Pediatr 103:979-984

11. Heymann MA, Rudolph AM, Silverman NH 1976 Closure of the ductus arteriosus in premature infants by inhibition of prostaglandin synthesis. N Engl J Med 295:530533

12. Flower RJ 1974 Drugs which inhibit prostaglandin biosynthesis. Pharmacol Rev 26:33-67

13. Hosmer DW, Lemeshow S 1989 Applied Logistic Regression. John Wiley \& Sons, New York

14. Firth J, Pickering D 1980 Timing of indomethacine therapy in persistent ductus. Lancet 2:144

15. Reller MD, Lorenz JM, Kotagal UR, Meyer RA, Kaplan S 1985 Hemodynamically significant persistent ductus arteriosus: an echocardiographic and clinical assessment of incidence, natural history, and outcome in very low birth weight infants maintained in negative fluid balance. Pediatr Cardiol 6:17-24

16. Hammerman C, Strates C, Valaitis S 1986 The silent ductus: its precursors and its aftermath. Pediatr Cardiol 7:121-127

17. Valdez-Crus LM, Dudell GG 1981 Specificity and accuracy of echocardiographic and clinical criteria for diagnosis of patent ductus arteriosus in fluid restricted infants. J Pediatr 98:298-305

18. Knight DB 1992 Patent ductus arteriosus: how important to which babies? Early Hum Dev 29:287-292

19. Murphy DJ, Corbet AJ, Long WA 199l The "natural" history of patent ductus arteriosus. Pediatr Res A 1456:245A

20. Yaffe SJ, Aranda JV 1991 Antipyretics. In: Yaffe SJ, Aranda JV (eds) Pediatric Pharmacology: Therapeutic Principles in Practice. WB Saunders, London, 2nd Ed, pp 335-350

21. Gerhard L 1975 Salicylate pharmacokinetics in the human neonate. In: Morselli PL, Garattini S, Sereni F (eds) Basic and Therapeutic Aspects of Perinatal Pharmacology. Raven Press, New York, pp 319-330

22. Rumack CM, Guggenheim MA, Rumack BH, Peterson RG, Johnson ML, Braithwaite WR 1981 Neonatal intracranial hemorrhage and maternal use of aspirin. Obstet Gynecol 58:52S-56S

23. Stuart MJ, Gross SJ, Elrad H, Graeber JE 1982 Effects of acetylsalicylic-acid ingestion on maternal and neonatal hemostasis. N Engl J Med 307:909-912

24. Karlowicz MG, White LE 1993 Severe intracranial hemorrhage in a term neonate associated with maternal acetylsalicylic acid ingestion. Clin Pediatr dec: 740:743 